\title{
The peculiarities of using the computer complex HC-psychotests in the process of psychodiagnosis of the level of development of future specialists' mental capacity
}

\author{
Hanna Varina*, and Svetlana Shevchenko \\ Bogdan Khmelnitsky Melitopol State Pedagogical University, Department of Psychology, 20 Hetmanska Str., Melitopol, 72300, Ukraine
}

\begin{abstract}
The IT revolution, which has embraced material production, social relations and the whole sphere of culture and education, is more and more contributing to solving the problem of automation of intellectual processes in science, technology and society, as well. This scientific article is devoted to the consideration of topical issues of introduction of the computer complex HC-psychotests in the process of psychological support of professional-personal development of the future specialist during the period of study in high school. The article also presents the analysis of the versatile possibilities of using the NSpsychotest in the paradigm of modern education. In this scientific article, the results of the screening study with the use of HC-psychotest in the process of psychodiagnosis of the level of development of mental capacity of future specialists are analyzed in detail. It is determined that the complex HC-psychotests allowed to give multilevel, multiparametric characteristics of the mental states of the respondents with the use of psychological, psychophysiological, physiological and social indicators. It has been proved that the introduction of computer psychodiagnostics has not only enriched the experimental base with appropriate methods that facilitate the implementation of various research strategies, but also combined empirical work into a single technological cycle.
\end{abstract}

\section{Introduction}

Nowadays, a new paradigm of cultural and educational space is being formed all over the world, within the framework of higher education, which is focused on joining the world information in educational and communicative space. Such a system leads to changes in the educational process, which must be in line with current technical capabilities and promote the harmonious adaptation of the future specialist in the information society. Computer technologies are aimed at increasing the efficiency of the educational process. Modern information computer technologies (ICT) are increasingly being introduced into various spheres of life, becoming an integral part of human existence and modern culture. ICTs enable automation of such information processes as: long-term and compact storaging, searching, processing, and producing of new, transmitting at any distance and presenting multimedia (text, spreadsheet, graphic, animated, audio and video) information.

The Concept of Higher Education Modernization has the important task of mastering the ICT skills by a modern person. Due to the change of professional activity dominant, it is necessary to prepare future specialists for various types of activities that are related to information processing, including the development of informatization tools. The introduction of modern information technology training has to be gradual: from the use of some elements of ICT training to the use of electronic textbooks [1].

The introduction of digital technologies in education creates a fundamentally new communicative environment, which requires a future specialist not only to have the ability to work with digital information, but also to possess "soft skills" - abilities to search and work with information, interpersonal communication, teamwork, flexible and creative thinking.

The global reformation process determines the relevance of introducing innovative ICT technologies into the educational environment. One of the directions of development of sustainable higher education is the development of a competitive specialist. In this regard, the four dominant competencies of a modern specialist are identified, which determine the success of professional tasks:

- Critical Thinking - Considered fundamental to the $21 \mathrm{st}$ century learning, critical thinking involves accessing, analysing and synthesising information in a way that helps learners evaluate ideas and make decisions.

- Communication - it is more important than ever to be able to communicate effectively and have the capacity to express thoughts clearly and persuasively in the workplace and public. Communication skills are embedded in information, media and ICT competencies

Corresponding author: varina hanna@mdpu.org.ua 
and will be more and more essential for employers and societal progress in the 21 st century.

- Collaboration - Collaboration and teamwork are highlighted as a critical area for development both in and outside of school. It is an essential skill for working in the 21 st century as 'local' takes on a new meaning.

- Creativity - Creativity and innovation will be in the forefront of our educational systems, like the ability to "break new ground", invoke fresh ways of thinking, put forth new ideas and solutions, pose unfamiliar questions, and arrive at unexpected answers will become crucial skills.

There are the following main areas of introduction of computer technology in the educational process: the use of computer technology as a means of learning; the use of computer technologies as a tool for learning about yourself and environment; consideration of computers and other modern information technology tools as objects of study; the use of new information technologies as a means of creative personal development; the use of computer technology as a means of automating the processes of monitoring, correction, testing and psychodiagnostics; organization of cooperation using information technology tools for the purpose of transferring and acquiring pedagogical experience, methodological and educational literature; the use of modern information technologies for the organization of intellectual leisure; intensification and improvement of the educational institution management and the process of education [2].

According to researchers, the number of students in our country who are able to use the computer and the Internet resources in various ways, has increased about 10 times during last 5 years. However, it is considered that they spend more time communicating on social networks and entertainment sites. At the same time, the cognitive motives, self-knowledge and self-development in the process of working with a computer and the Internet network are at about the 20th place. Simultaneously, the issue of training a competitive specialist, capable for constructively solving professional tasks in a changing living space and maintaining a high level of professional stability and efficiency, is urgent for modern higher education. Preparing young people for life and activity in a competitive and dynamic world requires professional and personal growth of the participants of the training process [3]. All this actualizes the problem of psychological support for the process of vocational training in vocational education institutions, its focus on students' personal and professional growth, the development of their ability to adapt quickly to the new conditions, to respond to new challenges, to develop democratic partnerships and interpersonal communication, in order to be an active participant of community.

The use of innovative ICTs in the process of psychological support for the professional development of future professionals is becoming a priority area of professional education.

\section{The main possibilities of using ICT in modern psychodiagnostics and educational practice}

The use of psychodiagnostics capabilities of modern computers to store compactly, to retrieve quickly and to display information clearly has certain benefits:

1. Versatility of equipment. A personal computer, by its functionality, can replace a whole set of devices and allow you to connect additional equipment if necessary. Computing standards allow the developer to focus on the methodical side of the test being created, which can be used on any personal computer.

2. Ability to generate tasks. In psychodiagnosis, the problem of adapting the respondent to stimulus material (e.g., to numerical tables) is an urgent one. The ability to generate test tasks allows you to create a large number of different tasks, to enter into the process of research trial (training) attempts and to use diagnostic techniques as simulators for the development of mental qualities.

3. Setting up experimental conditions. Managing the program settings opens wide opportunities for adjusting the conditions of the experiment. You can change the colour, shape and size of objects, exposure time and pauses, sound effects, etc. This makes it possible to create levels of complexity by adapting the methodology to a different contingent of respondents.

4. Test automation and standardization. The software implementation of the diagnostic methodology requires a specific algorithm that can provide a certain sequence of tests, depending on the results obtained. This creates test batteries and incentive simulators.

5. Ability to create new techniques using animation. The ability to depict objects in motion allows you to create new, more effective diagnostic and developmental techniques. The basis of such techniques is the modeling of dynamic processes and the involvement of the respondent directly in the development of the situation. Great prospects are opening up to the techniques implemented in the game form. The game (competitive) form of the test significantly changes the perception of the task, the respondent's motivation, attitude to the result.

6. Possibility of fixing additional parameters. In the computer implementation of diagnostic methods, when the entire process of testing is controlled by the program, it may be possible to fix additional parameters that extend the complex characteristics of the investigated mental phenomenon. For example, fixing the time of a single move in multi-pass manipulation and form tests allows you to study the dynamics of attention in the process of completing the task.

7. Online data processing. Test automation allows you to transfer all routine work to your computer, including mathematical and statistical processing of the data obtained. The high speed of computer calculation makes it possible to use methods that were previously little used due to their complexity (regression, variance, factor analysis, etc.) for data processing. Operative processing allows not only to evaluate the obtained results, but also to simulate other options for studying 
what is relevant in the study of compensatory mechanisms, mutual influence of mental processes, relationships in the group [4].

8. Enhanced results delivery. It is difficult to overestimate the value of presenting research results for their correct interpretation, and the personal computer provides virtually unlimited possibilities. The use of color, font, graphics, sound and animation allows to maximize the features of the results of the study. There are two types of automated psychodiagnostic findings. The first is aimed at for the respondent, and the second at the professional psychodiagnosis. These two types of interpretation should not be identical. Testimonials should primarily be formulated in a language of life psychology adapted for non-professional understanding. At the same time, "rapid interpretation is expected by the patient with impatience and increases his interest and motivational involvement in the survey" [4].

9. Increased standardization and validation. The use of computers helps to increase the level of standardization of conditions by equally instructing the respondents and by presenting tasks that do not depend on the sex, age, degree of attractiveness and mood of both the experimenter and the respondent. In addition, the privacy of an automated survey allows the respondent to be more forthright and natural during the research.

In addition to computer's benefits, the psychologist, according to Duke, is presented with qualitatively new opportunities to organize a computer psychodiagnostic examination.

1.Dynamic and polymodal stimulation. On the screen of a modern computer, it is possible to depict dynamic objects by means of computer graphics, which brings the model activity from the test execution to the real activity for which the test is intended [3]. The possibility of polymodal stimulation as a combination of its visual and auditory form is also of great importance.

2. Alternative order of test incentives. The simplest way of realizing this opportunity is to organize randomly the stimulus sequence. This principle also provides on adaptive testing, in which the sequence of presented tasks depends on the results of its responses to previous incentives. As a result, many of the respondent's tasks in the multistage testing process may not maintain the diagnostic capacity of the whole test. Due to the adaptive approach, it is possible to reduce significantly the complexity and time of testing.

3. Time as a factor in psychodiagnostics examination. With the help of a computer, a psychologist is able to adjust and set the required rate of psychodiagnostic testing. This rate can also be adjusted automatically, without the direct involvement of a psyhodiagnosis. On the other hand, time can serve as a diagnostic parameter, for example, as an indicator of the temporal dynamics of the answers to the test question.

4. Complex information processing algorithms. A wide range of various laborious procedures for calculating scales, indexes, auxiliary indicators, for carrying out diagnostic analysis related to finding precedents in a data bank, etc. has become possible.
5. Bank of psychodiagnostics data. Maintaining a data bank in which the results of psychodiagnostic research are accumulated can accelerate significantly the process of obtaining reliable, empirically substantiated test standards for the different contingents of respondents.

6. Game motivation. The "inclusion" of game motivation increases the attractiveness of the testing process and increases the reliability of the results. With the help of computer games, you can simulate certain activities. In the computer psychodiagnostic game, there is a possibility to combine verbal and nonverbal stimuli. On the one hand, a computer game is able to combine the functions of questionnaires and criterion-oriented performance tests. Complex KIM-88 - "complex game techniques" [5] - can serve as an example of this computer psychodiagnostics direction.

7.Display results. With the help of displaying information on modern computers it is possible to arrange the issuance of psychodiagnostic examination results on the screen or in the form of a profile of a person, graph or table, as well as the results of a survey towards the sample of respondents with the help of diagrams and histograms of the distribution of values of a given psychodiagnostic index. It is also possible to convert information using scaling methods and display the results of multivariate tests into two- and threedimensional pictures, which allow you to evaluate the groupings of the surveyed in the space of a multidimensional technique.

8. Intelligent interface. It is the possibility to receive various information, explanations, recommendations on preparation of psychodiagnostic examination and in the process of conducting it through a computer dialogue, as well as to draw a substantiated psycho-diagnostic conclusion in a comprehensive form [6].

\section{HC-psychotest is an innovative psychodiagnostic computer complex}

Rapid updating of information in all areas of knowledge challenges modern university with the most important tasks to prepare students who are able to be competent in modern society, who should manifest themselves in the following:

- the ability to adapt to rapidly changing conditions;

- to think independently and make decisions necessary for successful performance;

- to work competently with information, extract and process it, as well as to use effectively information resources, including global ones, to solve urgent problems of creating a sustainable education in a developing information space.

The priority perspectives of using innovative computer technologies in psychodiagnostic practice made it possible to open the laboratory of psychophysiological research at Bogdan Khmelnytsky Melitopol State Pedagogical University, which is equipped with the newest computer complex for psychotherapy. The relevance of using the HCpsychotest computer complex in the process of ensuring 
the sustainable development of higher education is due to the need to build an individual vector of professional and personal development of a future specialist taking into account individual psychophysiological indicators, level of performance, professional stability and professional orientation.

The use of the innovative HC-psychotest complex in the framework of modern higher education is focused on the implementation of the following functions: educational, research, diagnostic, correctional and developmental. The following are a number of significant advantages of using this computer complex in the modern educational process (Fig. 1):

1. The computer complex significantly expands the possibilities of providing educational information. The use of color, graphics, sound, all modern means of video technology allows you to recreate a real atmosphere of activity.

2. HC-psychotest can significantly increase student motivation to learn. Motivation is enhanced by the interconnection of theoretical foundations and the realities of practice.

3. The computer complex involves students in the educational process, contributes to the widest possible disclosure of their abilities, increases mental activity.

4. The use of this complex in the educational process increases the possibilities of setting educational tasks and managing the process of solving them. Computers allow you to build and analyze models of various objects, situations, phenomena.

5. This computer complex allows you to qualitatively monitor the activities of students, their activity and performance, while ensuring the flexibility of managing the educational process.

6. HC-psychotest allows students to diagnose the level of reflection and self-reflection, which is professionally important for the activities of future psychologists. The program allows students to visualize the result of their actions, to determine the stages of problem solution.

7. Based on the data obtained as a result of the integrated use of the HC-psychotest, it is possible to build an individual trajectory of training and professional development of future specialists.

As part of the implementation of the educational function and the actualization of the research potential of future specialists the HC-psychotest in the Laboratory of Psychophysiological Research is used in the following areas of activity:

- the use of graphic, video and audio elements for the integrated assimilation of educational material by future specialists and the implementation of an individual approach in the process of training students with special educational needs;

- the formation of practical skills of self-building an individual psychodiagnostics research program taking into account age-related characteristics and respondents' requests;

- in the process of studying psychological disciplines (for example, developmental psychology, experimental psychology, clinical psychology, political psychology, etc.), students solve practical competence-oriented tasks, cases, which ensures the internalization of theoretical knowledge and practical skills;

- in the process of studying the educational discipline "Psychodiagnostics", the development of practical skills in planning, conducting psycho-diagnostic research, collecting primary psycho-diagnostic data, compiling a protocol for psycho-diagnostic research, compiling a psychological portrait of a person and analyzing group processes based on the use of computer technology;

- in the process of studying the discipline "Mathematical Methods in Psychology", students use computer software to systematize and mathematically process psychodiagnostics data;

- the use of computer support in the process of monitoring the level of students' knowledge and analysis of educational effectiveness;

- in the process of implementing individual research activities, students have the opportunity to carry out virtual modeling of mental processes and phenomena, compiling a comprehensive psychodiagnostics program, planning an empirical study, implementing a program and strategy for empirical research - individual, intragroup or intergroup, mathematical processing of data, establishing correlation dependencies between variables, graphical and integrated presentation of the result own research.

In the process of implementing the diagnostic and correctional-developing function, the HC-psychotest is used in the following areas of activity:

- providing individual and group psychodiagnostics activities as a component of psychological support for students in the modern educational information environment;

- based on psychodiagnostics data, the construction of an individual vector of professional and personal development of future specialists in accordance with the requirements of the profession and student-centered strategy for the development of sustainable higher education;

- based on the results of psychodiagnostics, taking into account the individual psychophysiological characteristics of future specialists, the development of an individual strategy for teaching and developing students in order to improve the quality of professional education;

- development and testing of psychological correctional and developmental programs of the individual vector of professional and personal development of the personality.

Accordingly, the relevance of using this computer software is due to the following properties:

- the ability to optimize the continuous educational process of the individual (the ability to individually plan the place, time and pace of the educational process based on the results of psycho-diagnostic research and the construction of the vector of individual professional development);

- the opportunity to provide prospects for acquiring a new or related career in a short time;

- the adaptability of information technology training to individual psychophysiological characteristics of the student. 


\begin{tabular}{|c|}
\hline Psychophysiological diagnostics \\
\hline $\begin{array}{l}\text { aimed for identifying the individual features of the } \\
\text { course of nervous and mental processes at the time } \\
\text { of measurement amoung different age groups: } \\
\text { - psychomotor qualities (simple motor reaction time, } \\
\text { tepping, tremor, coordination, etc.); } \\
\text { - neurodynamic qualities (balance of nervous } \\
\text { processes, strength and endurance of the nervous } \\
\text { system, functional mobility of nervous processes, } \\
\text { features of interhemispheric asymmetry); } \\
\text { - qualities of attention (concentration and stability, } \\
\text { resistance to interference; selectivity, volume, } \\
\text { switching and distribution); } \\
\text { - individual qualities of perception, memory, } \\
\text { thinking; } \\
\text { - qualities of psychophysiological adaptability. } \\
\text { Examples of techniques: "Simple visual-motor } \\
\text { reaction" (PZMR), "Reaction to a moving object" } \\
\text { (WFD), "Critical fusion blinking frequency" } \\
\text { (CCSM), "Tapping test", "Contact coordination", }\end{array}$ \\
\hline $\begin{array}{l}\text { Psychodiagnosis of the whole spectrum of mental } \\
\text { phenomena }\end{array}$ \\
\hline ᄃ \\
\hline $\begin{array}{l}\text { - of mental processes: perception, memory, } \\
\text { attention, thinking ("Scales of devices", } \\
\text { "Identification of general concepts", "Memory for } \\
\text { numbers", etc.); } \\
\text { - individual mental qualities of temperament, } \\
\text { character, orientation and abilities (MMPI, Kettell } \\
\text { questionnaire, Eysenck techniques, Leonhard } \\
\text { questionnaire, Raven's Progressive Matrices, } \\
\text { Amthauer Intelligence Structure Test, etc.); } \\
\text { - mental states: short-term (activity, state of health, } \\
\text { mood, tension) and long-term (anxiety, depression, } \\
\text { aggressiveness, frustration) (method of assessment } \\
\text { of state of health, activity, mood (SAS), Luscher } \\
\text { test. etc.). }\end{array}$ \\
\hline
\end{tabular}

Fig. 1. The possibilities of using HC-psychotest in the area of higher education.

In the Laboratory of Psychophysiological Research, scientists use the following equipment of the HCPsychotest to solve questions of psychological support of future specialists:

1. Implement "Expert". A complete set that can be used in any psychophysiological study to assess the functional status and limitations of life on the basis of mental and physiological indicators, taking into account personal characteristics. The use of a polyregger allows to analyze the autonomic and emotional reactions during testing. This makes it possible to make a conclusion based on the physiological cost of the activity performed. A complete set of clinical questionnaires, techniques, and devices allow you to assess the level of adaptive capacity to neurodevelopmental stress. The state of the CNS and the regulation of the cardiovascular and respiratory systems are evaluated.
2. Implement "Candidate". An effective and easy-touse tool is used for career guidance and selection of candidates for vacant positions regardless of their work experience. The "Candidate" gives an opportunity to evaluate the level of expression of professionally important psycho-physiological qualities and professional competences, as well as to predict the further development of a specialist and to conduct indepth professional psychodiagnosis. An HR professional can use the results of research for understanding the strengths and weaknesses of a candidate, can make a decision about the feasibility of offering specific vacancies, can evaluate the limitations of future activity, or provide recommendations for developing an employee's potential.

3. Implement "Childhood". This set contains methods for psychological testing of children and adolescents. A block of projective techniques has been developed for preschoolers to assess their emotional state, level of school readiness, peculiarities of relationships in the family. For children of primary and secondary school age, a wide range of test materials is provided to study the features of cognitive functions, reactions to various activities, and the level of neuroticism. For high school students there is a career guidance block that helps to select the sphere of future activity according to psychotypes and personal qualities.

4. Implement "Sport" is a special set of tests, which allows to evaluate complexly important for sports activity psychophysiological and mental properties of the body: functional asymmetries of the person, personal anxiety, resistance to stress factors. Thanks to this equipment, the laboratory staff assesses the functional status of the athlete, his readiness to perform (play). Timely diagnosis of overtraining and detection of the initial stages of the disease is the main strategy of the set "Sport".

5. Implement "Start" is used in screening psychophysiological researches and gives an opportunity to investigate such issues as properties and states of personality; disorders of various mental functions; temperament and mental stability, interaction of personality and a group.

\section{The use of hardware devices in the process of psychodiagnostics}

For conducting psychophysiological tests, scientists at the Laboratory of Psychophysiological Research have the following hardware devices (Fig. 2):

1. Schulte Tables (Diagnosis of Attention Properties) is one of the most popular tests for assessing volume, distribution, and speed of switching attention. It is constantly used in professional selection in specialties requiring high concentration of attention. The test is performed on a special keyboard or using a touch screen.

2. Diagnosis of visual-motor reactions. Assessment of visual reactions has a key place in psychophysiology. The essence of the study is to measure the time of the motor response to the light stimulus. Analysis of the obtained results allows us to estimate the absolute time, 
stability, stability of the reaction, the probability of errors and failures. Accompanying indicators show the operator's readiness for work, the degree of his stress and fatigue.

3. Diagnosis of the strength of nervous processes. With the help of a tepping-test sensor, it is possible to determine lability and endurance of nervous processes, general human performance, resistance to monotonous activity, development of volitional personality qualities and other indicators of higher nervous activity in a matter of seconds.

4. Diagnosis of accuracy of movements. The coordinator is designed to evaluate tremor of the upper extremities at rest and during arbitrary movements.

5. Diagnosis of a pulse wave. The use of a miniature pulse-symmetric sensor allows you to determine the heart rate and $\mathrm{SpO} 2$ index.

6. The polyregistrator. To determine the level of adaptive capacity of the person for mental stress and to measure the physiological cost of the activity, a miniature device - a polyregistrator, was developed. It is capable of recording physiological parameters by means of three methods: ECG, oral pole muscle EMG, and pneumogram.

a)
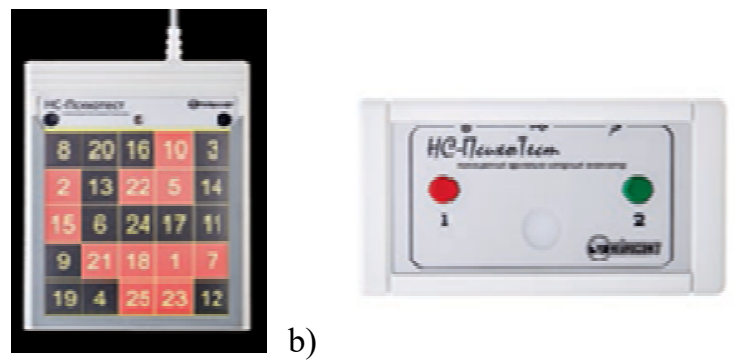

b)

c)
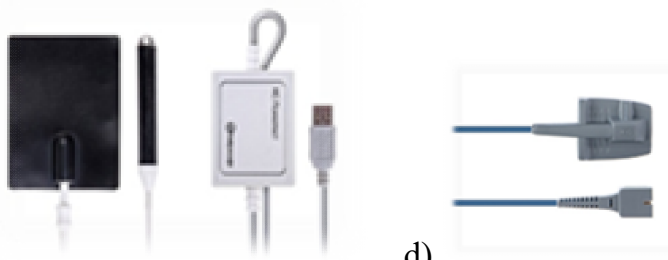

d)

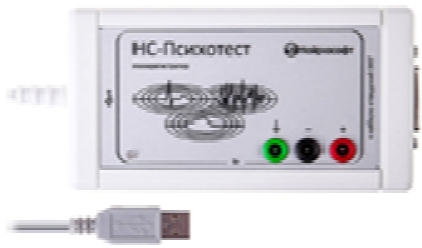

e)

Fig. 2. Hardware devices of the HC-psychotest (a) Schulte Tables; b) visual motor reaction recorder; c) tepping-test sensor; d) coordinator; e) polyregistrator).

\section{An empirical study of the level of development of mental abilities of a person using HC-psychotest}

As part of the scientific activity of the Laboratory of Psychophysiological Research in 2019, the staff conducted a screening computer psychodiagnostics study of the psychological features of students' mental performance. The ascertainment experiment, which was conducted during 2019 (Fig. 1), involved the first year students of Bogdan Khmelnitsky Melitopol State Pedagogical University (the total number of respondents was 162 people aged 17-22). The sample is representative. The sample was formed by the stratification method. All subjects were divided into subgroups of 10 people. In the diagnostic procedure, each student took an average of about one hour. A certificate of conformity No. PR.871-20 issued by the Ukrainian Scientific Certification Institute confirms reliability, validity, representativeness of psychodiagnostics techniques in the HC-psychotest computer complex. Also, using this computer complex, statistical processing of the obtained data was carried out. Computer processing of the results of psychodiagnostics studies, automatic execution of research protocols made it possible to significantly reduce the procedure for diagnosis and mathematical data processing.

According to scientists, mental work is characterized first of all by emotional tension. At the same time favorable emotional states, which systematically support the functional activity of other mental processes are the leading ones. According to the results of modern research, emotional processes directly regulate the mental capacity of the individual, maintain a certain level of mental activity [7]. Depending on the effect that the state of tension has on the efficiency of activity, states of operational and emotional tension are distinguished. Operating tensions are dominated by procedural motives, so it has a positive effect on the subject and maintains a high level of performance. Emotional tension is characterized by a pronounced negative affective color of behavior, the destruction of the motivational structure of mental activity, as a result, its effectiveness decreases [8]. It should be noted that in modern conditions the increase of emotional intensity and intensity of work, when various emotional stresses begin to act with great force on a person, many problems, related to the decrease of the level of intelligence, deterioration of emotional state and reduction of personality stress has arosed [8]. Personal emotions which are accumulated under the influence of powerful intellectual load reduce the level of respondent's mental capacity. Such stressful condition of the person is at the same time the cause and consequence of the occurrence of chronic fatigue in it [9].

The student's orientation as a subject to the knowledge of their own mental life is the epistemological aspect of the subject of psychology and an important educational aspect of the work of any teacher in the university, which is a real subject of pedagogical activity [10]. So, in ordering the theoretical achievements of scientists, based on the analysis and generalization of the scientific foundation, we have identified 4 basic criteria of mental capacity:

1) The cognitive component is the development of cognitive processes (the level of functioning of cognitive processes of the individual, the ability to perceive and 
select information according to the purpose of mental activity, to highlight the main, analyze, compare work, argue their thoughts and actions, memorize the necessary material);

2) The emotional component is an emotionalmotivational activity to intellectual actions (striving for cognition, initiative in performing intellectual tasks while maintaining emotional stability, activity and productivity of activity);

3) Personal component - subjectivity, objectivity, regulation of mental activity (the ability of the individuals to initiate, to perform mental activity independently and persistently, to evaluate it independently and critically and to take responsibility for their actions) [11];

4 The connective component is the performance of intellectual activity (a display of the completion of mental activity and orientation for the performance of intellectual activity).

On this basis, the mental capacity of the first-year student is considered by us as the integrative capacity of their personality to perform purposeful intellectual activity, which is ensured by the development of cognitive processes, emotional-motivational activity, as well as by their subjectivity, objectivity in activity.

Based on the results of theoretical and methodological analysis of the problem of research future specialists' mental capacity, we have developed a scheme of empirical research using the HC-psychotest:

1. In the first stage of the study, a comprehensive research program, focusing on the identified tasks of the work was developed.

2. At the second stage of the experiment, which was aimed to obtain data on the psychological characteristics of students' mental performance by its main components, a study was conducted at the Bogdan Khmelnitsky Melitopol State Pedagogical University.

In order to identify the psychological features of students' mental performance (focusing on certain basic criteria and components of mental performance), we applied the following group of techniques. To study the cognitive component of mental performance, which is presented by the development of students' cognitive processes, we have used the following techniques:

- The technique "Evaluation of attention selectivity" was chosen by us as a diagnostic material on the basis that a large amount of educational and scientific information requires students to be able to isolate objects on essential and insignificant grounds, to summarize them and to organize them.

- The state of optimum mental capacity is not possible without stability of attention. In order to identify the level of stability of students' attention in the course of the study, we used the method "Corrective test".

- The methodology "Analytical thinking research". The purpose of the study is to determine the level of development of analytic inductive thinking of students in a limited time. Analyticity is an important characteristic of thinking, in this case, we mean, the inductive thinking and the ability to operate (numbers).

The technique "The amount of short-term memory research", was aimed to determining the amount of students' short-term memory. The ability to retain and reproduce information quickly is of great importance for a successful university learning process.

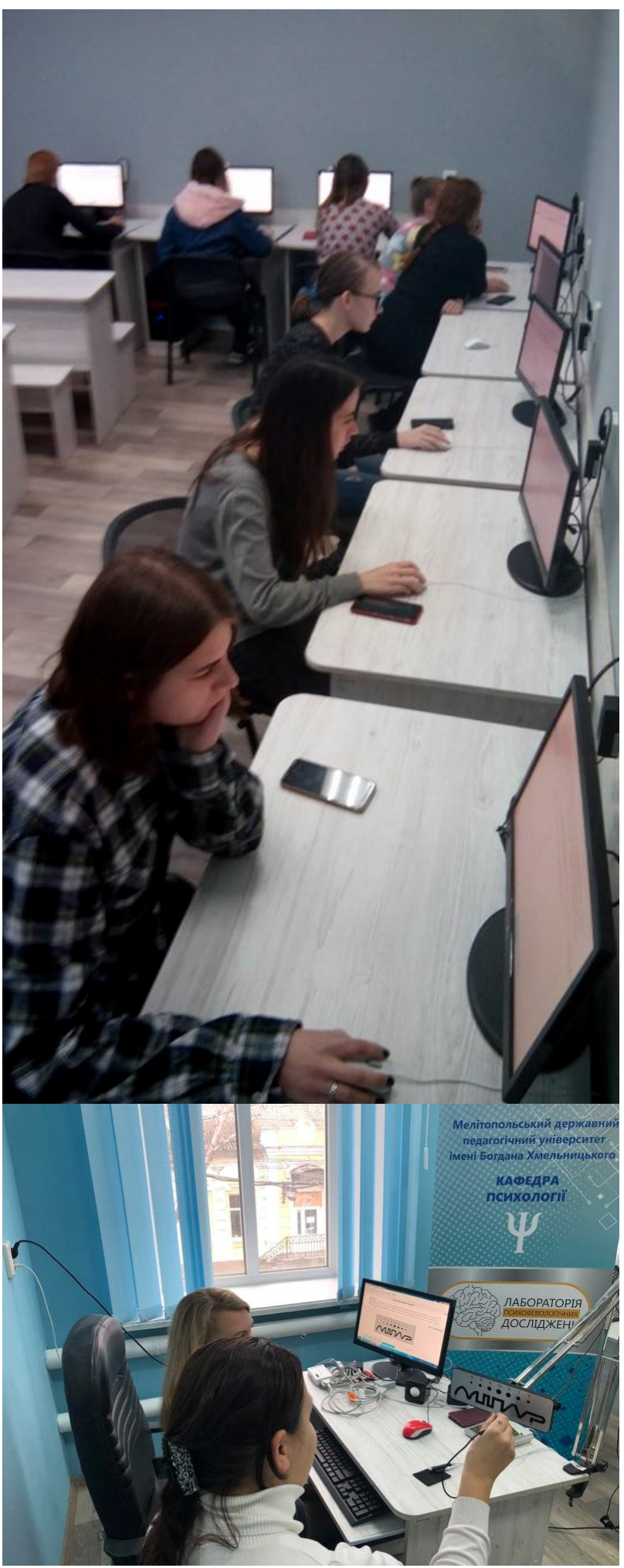

Fig. 3. Conducting a psychodiagnostics study using HCpsychotest.

In order to study the emotional component of mental performance, the method "Questionnaire DORS. Differentiated assessment of states of reduced performance (fatigue-monotony-supersaturation-stress), 
which is aimed at determining the degree of manifestation of each of the states of performance (fatigue-monotony-intersection-stress) was implemented. The development of these states leads not only to a decrease in mental performance, but also affects the qualitative characteristics of behavior and emotional coloring of experiences, which provokes significant changes in the motivational sphere of the individual [12].

In order to study the personality component of mental performance, we used the questionnaire "Autonomydependence of personality in educational activities" [13]. The questionnaire allows you to determine the ability to exercise autonomy through the mental performance, to evaluate critically person's intellectual activity, to execute it persistently.

The connective component of the mental performance of first-year students was studied by us on the basis of the analysis of their academic performance according to the results of the exam. At the same time, the indicators of absolute success, quality of knowledge, completeness of mental actions and levels of students' performance of intellectual activity were taken into account $[12,13]$.

According to the results of research of the cognitive component of mental capacity, indicators of the development of students' cognitive processes are revealed. The results obtained are presented in table 1 .

Table 1. Quantitative indicators (\%) of levels of development of the cognitive component first year students' mental capacity.

\begin{tabular}{|l|c|c|c|c|}
\hline $\begin{array}{c}\text { Cognitive } \\
\text { processes } \\
\text { development } \\
\text { levels }\end{array}$ & Attention & Attention & Analytical & $\begin{array}{c}\text { Short- } \\
\text { term } \\
\text { selectivity } \\
\text { attention } \\
\text { volume }\end{array}$ \\
\hline high & 29.47 & 9.12 & 17.90 & 11.93 \\
\hline medium & 43.85 & 52.63 & 61.05 & 69.12 \\
\hline low & 26.68 & 38.25 & 21.05 & 18.95 \\
\hline
\end{tabular}

Taking into account the indicators of the level of attention selectivity (method "Assessment of attention selectivity"), $43.85 \%$ of students has a medium level of attention selectivity development, which indicates their ability to select meaningful information and ignore irrelevant and indirect ones, to identify items on essential and non-essential features, to summarize them partially and to schematize them, this slightly reduces their level of intellectual and cognitive activity. $29.47 \%$ of the respondents are dominated by the high level of development. They have demonstrated the ability to distinguish objects by their essential properties, to summarize them and to schematize them, as well as their ability to find undivided images in the background. These are those first-year students who have accomplished the task quite well and have performed sufficiently intellectual exercises that require the use of this attention peculiarity.

But there were also students who have shown a low attention rate $(26.68 \%)$. They may not always be able to choose what is needed from the material they are offered, they can only allocate items on irrelevant grounds, and therefore will have some problems performing intellectual tasks. This result may be related to a state of intense emotional experience and external obstacles that led to the frustration of the respondent.

After processing the data of the method "Corrective test" results regarding the level of development of attention stability of first-year students were obtained (see table 2).

Table 2. Levels of development and indicators of students' mental capacity.

\begin{tabular}{|c|c|}
\hline Levels & Indicators \\
\hline High & $\begin{array}{l}\text { Students are able to distinguish subjects on essential } \\
\text { features, are able to generalize and schematize them, } \\
\text { maintain high intensity of attention for a long time } \\
\text { even in adverse conditions of activity, use logic in the } \\
\text { analysis and decision of intellectual tasks, } \\
\text { demonstrate prompt retention and reproduction of } \\
\text { information. } \\
\text { Fatigue has a short and temporary nature, motivated } \\
\text { to perform uninteresting mental activity, students do } \\
\text { not seek to stop it, no manifestations of stress. First- } \\
\text { year students are independent in their mental activity, } \\
\text { assess critically their intellectual activity, are } \\
\text { persistent in its implementation. They display special } \\
\text { creative abilities while exercising mental actions, } \\
\text { have the ability to complete thinking tasks. }\end{array}$ \\
\hline Medium & $\begin{array}{l}\text { Students are able to distinguish on both essential and } \\
\text { irrelevant grounds of subjects, they can partially } \\
\text { summarize and schematize them, while keeping } \\
\text { motivated attention on an interesting subject, use } \\
\text { logical conclusions in the conditions of attractiveness } \\
\text { of intellectual activity, more often memorize and } \\
\text { restore distinctive features. } \\
\text { They have a slight decrease in endurance, the } \\
\text { performance of intellectual activity may be } \\
\text { accompanied by a feeling of boredom and } \\
\text { drowsiness, prone to rejecting too subjectively } \\
\text { uninteresting activity, in response to increasing its } \\
\text { complexity they are experiencing stress. } \\
\text { First-year students cannot always properly and } \\
\text { deeply assess their intellectual abilities, and have } \\
\text { difficulty in overcoming obstacles during mental } \\
\text { activity. } \\
\text { They demonstrate productive performance of } \\
\text { intellectual tasks, partially completing mental } \\
\text { operations. }\end{array}$ \\
\hline Low & $\begin{array}{l}\text { First-year students distinguish subjects only by } \\
\text { insignificant features, they distract from bright } \\
\text { objects, have considerable difficulty in drawing } \\
\text { logical conclusions when solving intelligent tasks, } \\
\text { swindling to memorize and reproduce a small amount } \\
\text { of information. } \\
\text { They are in a state of exhaustion due to prolonged } \\
\text { and intense influence of mental stress, quite often } \\
\text { exhibit drowsiness and boredom, find a dominant } \\
\text { motivation to complete intellectual work and rest, } \\
\text { there are negative changes in mental and emotional } \\
\text { states. } \\
\text { Students perform mental activities based on the } \\
\text { attitudes of others, evaluate their intellectual abilities, } \\
\text { relying on their opinion and overcome difficulties in } \\
\text { carrying out mental activities under their control. } \\
\text { They do not complete mental actions and are only } \\
\text { prone to reproduction while performing intellectual } \\
\text { activity. }\end{array}$ \\
\hline
\end{tabular}

The majority of students (52.63\%) showed a medium level of development of attention stability. They 
demonstrated the ability to keep it on a certain object, not to distract and not weaken it, but only when they are motivated and depend on the peculiarities of these objects (their attractiveness, brightness, curiosity for the subject). $38.25 \%$ of first-year students are dominated by low levels of attention development. They are tending to be distracted by other bright objects and change the purposeful activity, also they have certain problems in performing long, monotonous and difficult mental activity.

$9.12 \%$ of respondents are characterized by a high level of development of this attribute of attention, which indicates their ability to maintain a high intensity of attention for a long time, awareness of the importance of performing mental tasks, even in the presence of unfavorable working conditions, which is a basic background for intellectual productivity, and mental activity of students.

The results of the study by the method of "Investigating the analytic thinking" demonstrated that most first-year students $(61.05 \%)$ were diagnosed with a medium or satisfactory level of thinking analytic development. This indicates that they have developed the ability to use logical conclusions in terms of attractiveness and importance of intellectual activity, which allows them to perform successfully various activities, including mental.

In $21.05 \%$ of the respondents revealed a high level of this indicator, which is identified in the effective solution of mental problems with the help of logical conclusions, due to high ability of planning their activities, finding specific solutions to problems and the desire to search. These first-year students while exercising mental activity are able to break down information into separate components, to implement a comprehensive analysis of these components, as well as to identify information as a whole, to identify several options for its solution, analyses and evaluate each option. They solve complex problems more efficiently and faster than others, make logical conclusions, even in the absence of information, look at the problem from different points of view, so they are able to find the best solution.

Unfortunately, in the course of the research, low results were also found on this characteristic of thinking. In particular, $16.14 \%$ showed low and $1.76 \%$ showed a very low level of analytical thinking. They have significant difficulties in drawing logical conclusions when solving intellectual problems, they have not developed the ability to theorize, find causal relationships between phenomena, have no skills to make reliable assumptions about the most likely options for the development of events, can't restore effectively the necessary information through logical conclusions which affects the process of mental activity and its effectiveness.

The technique "Study of the short-term memory amount", which is aimed at determining the amount of students' short-term memory, showed the following results (see Table 2). Most first-year students (69.12\%) demonstrated a medium level of short-term memory, showing the ability to memorize and reproduce only relevant, interesting, necessary information and expressive features of individual subjects. Therefore, they have some potential for successful mental activity because the modern learning process is associated with a high mental load.

$11.93 \%$ of respondents are characterized by a high level of this type of memory. They are able to memorize a large amount of intellectual information, retain and reproduce it promptly, which provides high mental performance. Despite this, $18.95 \%$ of students showed poor results on this indicator. Respondents, who have difficulty in perceiving information, memorizing and reproducing perceived material, being motivated to memorize only a small amount of it, require systematic training of memory.

Qualitative and quantitative analysis of the emotional component of mental performance, study especially the degree of manifestation of each of the states of performance (fatigue-monotony-satiety-stress) according to the method "Questionnaire DORS. Differentiated assessment of states of reduced performance (fatiguemonotony-supersaturation-stress)" determined that a moderate level of fatigue was found among $84.91 \%$ of students; they have such a state of performance only partially present in the process of their mental work, they have a slight decrease in endurance, the presence of some minor errors in the performance of intellectual activity, which almost does not affect its effectiveness.

At the same time, $11.58 \%$ of respondents showed a low level of this indicator. They do not feel exhausted, because the exhaustion in the performance of mental work is temporary and short-term in nature, performing it without significant mistakes, thus having considerable potential for processing mental tasks.

The study found that $3.51 \%$ of first-year students have a pronounced level of fatigue, they are in a state of exhaustion, performing mental exercises and developing tasks due to the prolonged and intense impact of mental stress, with a dominant motivation to complete intellectual work and rest. They observe a decline in labor productivity, a significant increase in mental activity errors, an increase in neuro-emotional excitability, and a decrease in overall endurance.

The moderate level of monotony that occurs in situations of monotonous work with frequent repetition of stereotypical actions and is accompanied by a feeling of boredom, drowsiness and dominant motivation to change activity prevails in $68.07 \%$ of the researches. $18.95 \%$ of students are characterized by low levels of this condition. They only sometimes have a slight decrease in activity with overall involvement, even in monotonous mental work. For $12.98 \%$ of first-year students there is a pronounced level of monotony, which is displayed in a decrease in attention and overall ability to willpower, and sometimes a refusal to perform mental activity.

It was found out that $71.93 \%$ of students were diagnosed with a moderate level of manifestation of this condition according to the index of mental satiation. They are sometimes inclined to reject too much subjectively uninteresting activity, to change a given stereotype of performing intelligent actions. $28.07 \%$ of respondents showed a low level of saturation. They are 
able to perceive uninteresting mental activity without the desire to stop it.

The state of tension and stress (as one of the indicators of reduced working capacity) is partially available for most students $(77.89 \%$ found a moderate level of manifestation of this condition). This suggests that they are partly tend to be stressed, which may be manifested in the overall mobilization of psychological and energy resources that is developed in response to increased complexity or subjective significance of the activity. Only $22.11 \%$ of first-year students were found to have low rates of stress. They do not have significant changes in their mental and emotional states, which ensures optimal mental performance.

According to the results of diagnosing the peculiarities of the personality component of mental capacity the questionnaire "Autonomy-dependence of personality in educational activities" found out that students with a high level of independence $(75.09 \%)$, criticality $(18.25 \%)$ and perseverance $(23.16 \%)$ when performing their intellectual tasks demonstrate independence in defining the goals and objectives of mental activity, the ability to evaluate objectively and to implement obstinately. They have the habit and ability to analyze the reasons of success and failure at work, stand alone in defining their goals and objectives, and to choose the right ways to solve them. These traits are necessary for successful study in a higher education institution.

The study found out that students with a medium level of development of independence (22.11\%), criticality $(70.53 \%)$ and perseverance $(69.47 \%)$ carry out new mental activity only with support, cannot always evaluate their intellectual abilities, have difficulties in overcoming obstacles in solving intellectual problems.

Respondents with a low level of independence $(2.80 \%)$, criticality $(11.23 \%)$ and perseverance $(7.37 \%)$ perform mental activity with teacher's support, overcome difficulties in carrying out mental actions only under their control, evaluate own intellectual potential based on the opinion of others. That is why, they perform mental activity, focusing on the attitudes of others, assessing their intellectual capacity, relying on their opinion and overcoming difficulties in carrying out mental activities under their control.

Studying the connective component of the mental performance of the first-year students, we analyzed the indicators of their academic success according to the results of the winter exam.

In order to obtain the average indicators for a given component, the results of the whole sample, rather than of individual academic groups, were taken into account, in terms of absolute success, quality of knowledge and levels of intellectual activity performance. As a result of the empirical data processing, it is established that the student's absolute success rate is $92.93 \%$ of the total number of respondents. This is due to the fact that not all first-year students (namely $7.07 \%$ ) received satisfactory grades during the session. They have training materials at the level of individual fragments, have difficulty completing their intellectual activity. The average quality of students' knowledge is $61.84 \%$. This percentage of research is combined to make the session "excellent" and "excellent" and "good".

Analyzing the performance levels of intellectual activity (according to the results of the session), it was found that $4.95 \%$ of students showed high performance, showing special creative abilities in the exercise of intellectual activity, the ability to independently acquire knowledge, to complete the tasks, without the help of a teacher, to find and process the necessary information; the ability to use the acquired knowledge, to reveal their own slopes independently.

The analysis showed that $56.89 \%$ of first-year students demonstrated medium performance, concluding the session on the assessment of "excellent" and "good". They are not always fully capable to perform mental actions and show productive performance of intellectual tasks, and they possess freely the amount of educational material, correcting mistakes themselves, the number of which is small, able to generalize, systematize information under the guidance of the teacher.

According to the results of the winter examinations students with low level of performance $(38.16 \%)$ were identified. This group of respondents, exercising the intellectual activity, is only prone to reproduce the educational material, expressing knowledge and understanding only of the basic provisions, with the help of a teacher they are able to analyze mental actions and correct mistakes, among which there are a considerable number of material ones.

The results of our theoretical and experimental achievements have allowed us to determine the levels of development (low, medium and high) and the qualitative indicators of mental performance, which were substantiated on the basis of the above criteria (Table 2).

Thus, assessing the peculiarities of students' mental performance during the period of adaptation to higher education, it was found out that the majority of students dominate the medium level of development of this ability by the identified components (average level of development of stability and selectivity of attention, analytical thinking, amount of short-term memory, monotony, satiety, stress, and average indicators of development of independence, criticality and perseverance in the exercise of mental activity).

The first-year students have the ability to distinguish subjects on both essential and insignificant grounds, have partially developed skills to generalize and schematize them, when being motivated they retain their attention on an interesting subject, use logical conclusions in terms of attractiveness of intellectual activity, more often they remember and refresh the items peculiarities.

The respondents were diagnosed with a slight decrease in endurance, errors in the performance of work. In situations of monotonous work with frequent repetition of stereotyped actions, they are prone to feel boredom, drowsiness with dominant motivation to change activity. Most students are sometimes inclined to reject too much subjectively uninteresting activity, which is manifested in a marked refusal of activity, or to make changes to a given stereotype of performance in a 
situation of increased complexity or subjective significance of mental activity.

At the same time, first-year students are diagnosed with a medium level of development of such personality traits as: independence, criticality and perseverance in performing intellectual actions. In a situation where new mental activity is necessary, they are able to perform it with the support, they cannot always correctly and deeply assess their intellectual abilities and behavior, they seek better ways to perform successfully their mental work, they have difficulty in overcoming obstacles in their mental activity.

Most respondents showed a medium level of intellectual activity performance, having completed the session on the assessment of "excellent" and "good", by partially completing mental operations, demonstrating the productive performance of mental tasks, while having a sufficient amount of educational material, by correcting a small number of errors with the help of a teacher.

\section{Conclusion}

Thus, in general, first-year students have a medium level of development of mental capacity for all the selected components (cognitive, emotional, personal and connective), sufficient potential to successfully master the knowledge of high quality and productivity of mental work. In spite of this, quite low indicators of mental capacity for the identified components have been found out, which requires the development of a system of measures for improving the mental capacity of first year students.

In the process of screening, the scientists have identified the following advantages of using a computer complex of HC-psychotests:

1. The complex allowed to give multi-level and multiparametric characteristics of the mental states of the respondents using psychological, psychophysiological, physiological and social indicators.

2. The HC-psychotests realizes the possibility of registration and analysis of autonomic and emotional reactions during psychological and psychophysiological testing, which allows to evaluate the physiological cost of the activity, the level of adaptive capacity for loads with neuro-psychic stress (NPS), to predict more accurately the availability of NPS loads, with, in real working conditions.

3. Population standards by all methods are stored in the base of standards. With the help of the editor of the database of norms it is possible to view and edit them, taking into account data of own researches. It is possible to use multiple test bases for testing (for example, standard ones, supplied with the program, and your own) and a quick transition from one rule base to another.

4. The software of the complex allows to print paper test forms (incentive material and registration forms of testing) for the test-questionnaires, as well as to perform automated analysis using a scanner (editable) filled in by the test forms.
5. The program realizes the possibility of creating new testing methods according to the tasks of the study through a flexible system of settings of test parameters and with the help of the built-in editor of tests and templates of testing. The test editor allows you to specify instructions for completing the test tasks that appear on the monitor screen before testing, to create questionnaire texts and to establish principles for processing the data obtained. The template editor allows you to form a sequence of tests.

6. Initial test data such as physiological signal recordings, stimulus responses, selected response options, and more are available for viewing, processing and exporting to standard statistical processing programs to calculate specific results not provided by the mathematical apparatus of the program.

7. In the process of psycho-diagnostics of mental capacity, we conducted group testing (up to 10 people at a time) in an automated mode. This increased the capacity of the complex and simplified the task of examining a large group of people.

8. For each respondent registered in the database, upon receipt of results by any method, an individual norm database is formed. So, if an individual base is formed, the next test of this respondent program will "tell" whether the next result is typical for a person, regardless of population norms, which may differ from the individual ones.

Thanks to the use of the technology of HCpsychotests, computer psychodiagnostics techniques become the most attractive and widespread tools of psychologists conducting examinations in various fields and in the system of training of future specialists in higher education as well. Their development involves the creation of systems that construct a diagnostic conclusion based on the examination of a specific subject in the form of a coherent and consistent text, which quite fully reflects the psychological parameters measured by the test. In this case, the automated conclusion is individual for each respondent, and does not display some "average" characteristics and parameters that correspond to a certain contingent of people. Thus, computerization of psychodiagnostics techniques has a positive effect on improving the quality and reducing the cost of psychodiagnostics work in the process of psychological support for the professional development of a future specialist. The use of the HCpsychotests in the field of education helps to expand the professional horizons and iterate the practical and theoretical experience of the student; training of graduates to live in the information society; implementing a social order that focuses on global informatization processes.

\section{References}

1. V. Osadchyi, K. Osadcha, V. Eremeev, The model of the intelligence system for the analysis of qualifications frameworks of European countries. International Journal of Computing 16(3), 133-142 (2017) 
2. V.S. Kruglik, V.V. Osadchyi. Formation of competence in the field of programming for future software engineers. Integration of Education 23(4), 587-606 (2019)

3. Y. Chen, A. Johri, H. Rangwala, Running out of STEM: A comparative study across STEM majors of college students at-risk of dropping out early, in Proceedings of the 8th international conference on learning analytics and knowledge, ACM, 2018, pp. 270-279

4. L. Ali, M. Hatala, D. Gašević, J. Jovanovic, A qualitative evolution of a learning analytics tool. Computers \& Education 58, 470-489 (2012)

5. T. Liao, C, Keng, Finding a psychological recovery strategy by online consumer experiences. Computers in Human Behavior 29(4), 1849-1861 (2013)

6. T. Buccheri, The Importance of Psychodiagnostic Evaluation to Structure Effective and Integrated Prevention Program: A Preliminary Sicilian Study. J. Obes. Weight Loss Ther. 3(5) (2013)

7. R. Ferguson, Learning analytics: Drivers, developments and challenges. International Journal of Technology Enhanced Learning 4(5/6), 304-317 (2012)

8. V.V. Delibalt, A.V. Degtyaryov, E.G. Dozortseva, R.V. Chirkina, N.V. Dvoryanchikov, V.A. Pimonov, M.G. Debolsky, D.A. Malkin, Evaluation of cognitive functions, personality and regulatory sphere in minors with deviant and delinquent behavior within the authority of the psychological, medical and educational committee. International Journal of Cognitive Research in Science, Engineering and Education 5(2), 107-118 (2017)

9. R.A. Vidourek, M. Burbage, Positive mental health and mental health stigma: A qualitative study assessing student attitudes. Mental Health \& Prevention 13, 1-6 (2019)

10. I.S. Aron, Social Situation of Development: Structural and Content Analysis of the Concept in the Context of Professional Self-Determination. Cultural-Historical Psychology 4, 53-58 (2013)

11. A.S. Young, M.R. Meers, A.T. Vesco, A.M. Seidenfeld, L.E. Arnold, M.A. Fristad, Predicting Therapeutic Effects of Psychodiagnostic Assessment Among Children and Adolescents Participating in Randomized Controlled Trials. Journal of Clinical Child \& Adolescent Psychology 48, 1-12 (2019)

12. M.W. Acklin, Psychodiagnosis of Personality Structure: Psychotic Personality Organization. Journal of Personality Assessment 58(3), 454-463 (1992)

13. M. Guarcello, R. Levine, J. Beemer, J. Frazee, M. Laumakis, A. Schellenberg, Balancing student success: assessing supplemental instruction through coarsened exact matching. Technology, Knowledge and Learning 22(3), 335-352 (2017) 